\section{Hocheffiziente photolabile Schutzgruppen mit intramolekularem Energietransfer**}

\author{
Dominik Wöll, Julia Smirnova, Wolfgang Pfleiderer \\ und Ulrich E. Steiner*
}

DNA-Chips werden in stark zunehmendem Maße in der Genomanalytik eingesetzt. ${ }^{[1]}$ Inzwischen können hochdichte DNA-Chips mit einigen Hunderttausend verschiedener Punkte (Spots) pro $\mathrm{cm}^{2}$ hergestellt werden. ${ }^{[2]}$ Damit lässt sich im Prinzip das gesamte menschliche Genom erfassen ${ }^{[3]}$ Solch hohe Punktdichten erzielt man durch eine photolithographische In-situ-DNA-Chip-Synthese. Hierbei werden die Oligonucleotide schrittweise aus geschützten Nucleosidphosphitamiden mit Photolithographie durch Masken ${ }^{[4]}$ oder Mikrospiegel-Anordnungen $^{[5]}$ räumlich definiert aufgebaut. Diese Technik erfordert photolabile Schutzgruppen ${ }^{[6]}$ mit hoher Lichtempfindlichkeit, die ihr Substrat (in diesem Fall ein Nucleotid) in nahezu quantitativen Ausbeuten freisetzen. Eine charakteristische Maßzahl für die Lichtempfindlichkeit stellt das Produkt $\varepsilon \varphi$ aus Absorptionskoeffizient und Quan-

[*] D. Wöll, Dr. J. Smirnova, Prof. Dr. Dr. W. Pfleiderer, Prof. Dr. U. E. Steiner

Fachbereich Chemie, Universität Konstanz Universitätstraße 10, 78464 Konstanz (Deutschland) Fax: (+49) 7531-88-3014

E-mail: ulrich.steiner@uni-konstanz.de

[***] Die Autoren danken der Deutschen Forschungsgemeinschaft für finanzielle Unterstützung (Projekt Ste 283/7-1) und Firma NimbleGen $\mathrm{GmbH}$, Waldkraiburg, für die Durchführung von Chipexperimenten. tenausbeute dar; es erreicht bei den bisher für die DNAChip-Synthese üblichen Schutzgruppen nur mäßige Werte. Die [( $\alpha$-Methyl-2-nitropiperonyl)oxy]carbonyl(MeNPOC)Schutzgruppe ${ }^{[7]}$ weist bei den praktisch eingesetzten Wellenlängen (vorzugsweise wird die Quecksilberlinie bei $\lambda=$ $366 \mathrm{~nm}$ verwendet) zwar einen akzeptablen Absorptionskoeffizienten auf $\left(\varepsilon_{\mathrm{MeOH}, 366 \mathrm{~nm}} \approx 2500 \mathrm{M}^{-1} \mathrm{~cm}^{-1}\right)$, jedoch ist die Quantenausbeute für die Entschützung sehr gering (3\% in $\mathrm{MeOH}) \cdot{ }^{[8]}$ Im Unterschied dazu zeigt die 2-(2-Nitrophenyl)propoxycarbonyl(NPPOC)-Schutzgruppe, ${ }^{[9]}$ deren Reaktion in Schema 1 dargestellt ist, ${ }^{[10]}$ eine wesentlich höhere

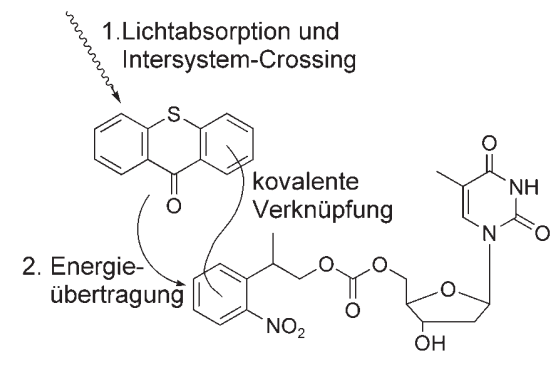

$$
\begin{aligned}
& \text { 3. H-Transfer } \\
& \text { 4. } \beta \text {-Eliminierung } \\
& \text { und Fragmen- } \\
& \text { tierung }
\end{aligned}
$$

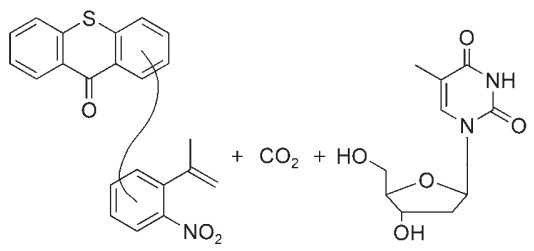

Schema 1. Die intramolekular sensibilisierte Photoabspaltung einer Schutzgruppe vom Typ der Verbindung 1.

Quantenausbeute (41\% in $\mathrm{MeOH})$, allerdings absorbiert sie das Licht viel schlechter $\left(\varepsilon_{\mathrm{MeOH}, 366 \mathrm{~nm}} \approx 250 \mathrm{M}^{-1} \mathrm{~cm}^{-1}\right)$. Sowohl bei MeNPOC als auch bei NPPOC ergeben sich dadurch lange Belichtungszeiten, während deren unerwünschte Photoreaktionen stattfinden können.

Die Lichtempfindlichkeit schwach absorbierender photolabiler Schutzgruppen lässt sich prinzipiell mithilfe von Sensibilisatoren erhöhen. ${ }^{[11,12]}$ Durch Triplett-Sensibilisierung von NPPOC mit Thioxanthon gelang eine beachtliche Steigerung der Empfindlichkeit der Schutzgruppenabspaltung in Lösung wie auch auf einer Chipoberfläche. ${ }^{[11]}$ Die Lichtenergie wird hierbei größtenteils vom Sensibilisator aufgenommen und kann auf die Schutzgruppe übertragen werden, falls ein angeregtes Sensibilisatormolekül während seiner Lebensdauer auf ein Schutzgruppenmolekül trifft. Die Diffusion ist bei diesem intermolekularen Prozess der begrenzende Faktor für eine effektive Energieübertragung. Intramolekulare Triplett-Triplett-Energieübertragungen sind verschiedentlich beschrieben worden. ${ }^{[13]}$ Hier stellen wir neue photolabile Schutzgruppen vor, die das Prinzip der intramolekularen Sensibilisierung nutzen: Ein Sensibilisator wird kovalent mit der Schutzgruppe verknüpft, sodass eine von der 
Diffusion unabhängige, höhere Lichtempfindlichkeit resultiert. $^{[14]}$ Kürzlich berichtete auch die Gruppe von Corrie ${ }^{[15]}$ über die Photolyseverstärkung einer photolabilen Verbindung durch eine kovalent angeknüpfte Antenneneinheit.

Details der Synthesen der in Schema 2 dargestellten Schutzgruppen finden sich in Lit. [16]. o-Nitrophenyl- und Thioxanthon-Chromophor wurden an unterschiedlichen
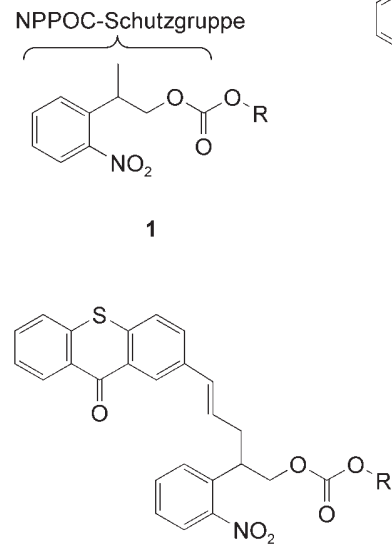<smiles>[R]OC(=O)OCC(CCc1ccc2sc3ccccc3c(=O)c2c1)c1ccccc1[N+](=O)[O-]</smiles>

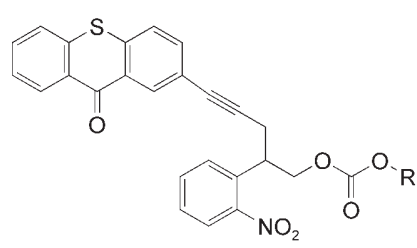

2

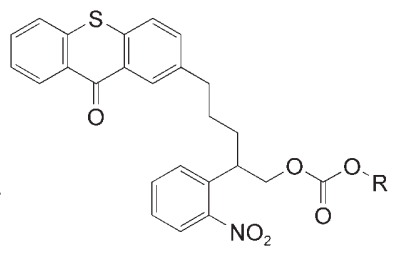<smiles>[R]OC(=O)OCC(C)c1cc(-c2ccc3sc4ccccc4c(=O)c3c2)ccc1[N+](=O)[O-]</smiles><smiles>[R]OC(=O)OCC(C)c1ccc(C(=O)Oc2ccc3sc4ccccc4c(=O)c3c2)cc1[N+](=O)[O-]</smiles>

Schema 2. Übersicht zu den diskutierten Verbindungen. $R=5^{\prime}-O$-Thymidinyl.

Stellen über verschiedene Linker verknüpft; mit Ausnahme von Verbindung 7 geschah dies durch C-C-Kupplungen (Sonogashira-, Heck-, Suzuki-Kupplungen). Zur Synthese der über eine Kohlensäurediester-Funktion geschützten Thymidine $(\mathrm{R}=5$ '-O-Thymidinyl) wurden die Alkohole $(\mathrm{R}=\mathrm{H})$ zunächst mit Phosgen oder Phosgen-Ersatzstoffen und dann mit Thymidin umgesetzt.

Abbildung 1 zeigt die Absorptionsspektren der geschützten Thymidine. Mit Ausnahme von Verbindung 6 wird die Absorption im Wesentlichen durch den Thioxanthonteil bestimmt. Für die 2-Vinyl- und die 2-Ethinyl-Derivate bewirkt die Substitution signifikante Änderungen in der zweiten Absorptionsbande gegenüber dem freien Thioxanthon. Der NPPOC-Chromophor absorbiert im gezeigten Bereich nur relativ schwach, Thymidin so gut wie nicht. 6 ist die einzige Verbindung, bei der zwischen dem Thioxanthon- und $o$-Nitrophenyl-Chromophor eine unmittelbare elektronische Kopplung der $\pi$-Elektronensysteme besteht. Die bichromophoren Schutzgruppen 2-5 und $\mathbf{7}$ haben aufgrund ihres

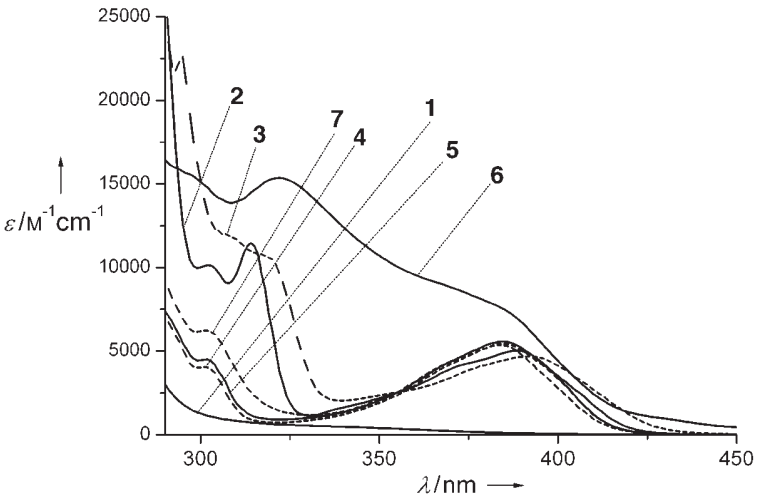

Abbildung 1. Absorptionsspektren der Verbindungen 1-7 in Methanol.

Thioxanthonteils bei $366 \mathrm{~nm}$ einen etwa 15 fach, Verbindung 6 sogar einen etwa 35 fach höheren Absorptionskoeffizienten als die NPPOC-Schutzgruppe. Bei gleicher Quantenausbeute ergäbe sich also eine ca. 15- bzw. 35-mal höhere Lichtempfindlichkeit. Um dies zu verifizieren, wurden die mit unseren neuen Schutzgruppen geschützten Thymidine kontinuierlich bestrahlt (Bedingungen: 0.05 bis $0.15 \mathrm{~mm}$ in Methanol, Lichtintensität ca. $2 \times 10^{-8}$ Einstein $\mathrm{cm}^{-2} \mathrm{~s}^{-1}$, bestimmt mit Azobenzol-Aktinometrie ${ }^{[17]}$ ); die Produkte wurden nach verschiedenen Bestrahlungszeiten durch HPLC getrennt und UV/Vis-spektroskopisch analysiert. Die Abnahme der Konzentration der Ausgangsverbindung wird durch die Differentialgleichung (1) beschrieben. Darin bedeuten $I_{0}$ die

$\frac{d c}{d t}=-I_{0} \frac{F d}{V} \frac{\left(1-10^{-A(t)}\right)}{A(t)} \varepsilon c(t) \phi$

molare Photonenflussdichte, $F$ die bestrahlte Küvettenfläche, $d$ die Schichtdicke, $V$ das Lösungsvolumen, $A(t)$ die Absorbanz der Lösung nach der Bestrahlungszeit $t, \varepsilon$ den Absorptionskoeffizienten der Schutzgruppenverbindung, $c$ deren Konzentration und $\phi$ die Quantenausbeute der Entschützungsreaktion. Eine Anpassung der durch numerische Integration von Gleichung (1) erhaltenen Funktion $c(t)$ an die gemessene Abhängigkeit der Konzentration der Ausgangsverbindung von der Bestrahlungszeit (Abbildung 2a) liefert die Quantenausbeute $\phi$.

Tabelle 1 zeigt, dass die Quantenausbeuten für die bichromophoren Schutzgruppen etwas - allerdings nicht gravierend - niedriger sind als für die NPPOC-Schutzgruppe. Diese Verminderung der Quantenausbeute wird durch die Erhöhung des Absorptionskoeffizienten um ein Vielfaches überkompensiert, sodass die Lichtempfindlichkeit bis $\mathrm{zu}$ 21 fach bei $366 \mathrm{~nm}$ bzw. 25 fach bei $405 \mathrm{~nm}$ gesteigert ist. Die Ausbeuten an entschütztem Substrat liegen für die Verbindungen 4-7 zwischen 66 und 92\% (Tabelle 1), sollten aber durch weitere Optimierung der Reaktionsbedingungen noch zu verbessern sein. Die schlechten Thymidin-Ausbeuten bei $\mathbf{2}$ und $\mathbf{3}$ lassen sich auf spezifische Nebenreaktionen zurückführen. Bei Verbindung 3 erfolgt hauptsächlich eine trans-cisIsomerisierung, bei Verbindung 2 fällt ein Umlagerungsprodukt gleicher Molmasse an, dessen Konstitution jedoch nicht aufgeklärt wurde. 

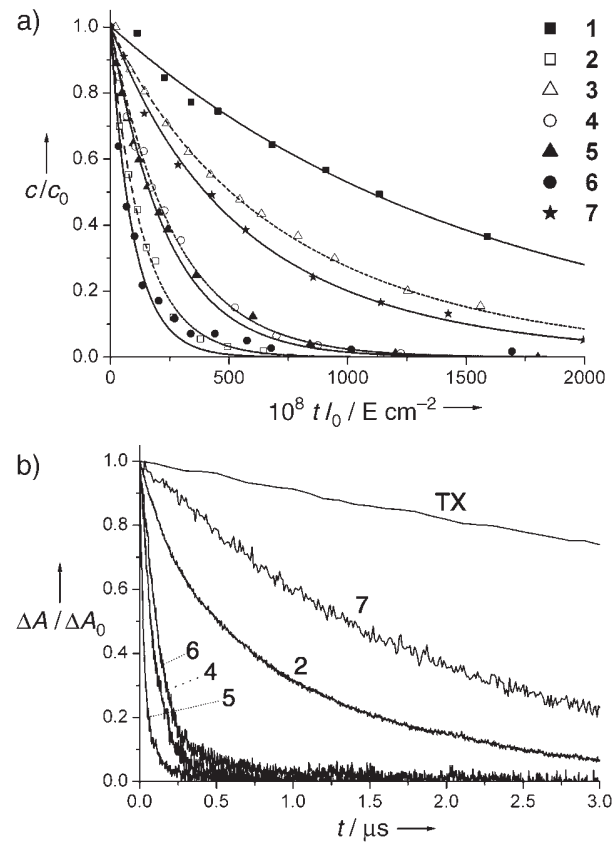

Abbildung 2. a) Reaktionskinetik des Abbaus der geschützten Thymidine 1-7 bei kontinuierlicher Bestrahlung mit $\lambda=366 \mathrm{~nm}$. Die absolute Lichtintensität $I_{0}$ betrug ca. $2 \times 10^{-8}$ Einstein $\mathrm{cm}^{-2} \mathrm{~s}^{-1}$. Die Datenpunkte wurden durch Integration der nach den jeweiligen Belichtungszeiten beobachteten HPLC-Peaks erhalten; mit Gleichung (1) wurden Kurven an die Messwerte angepasst. b) Abklingkinetik des Thioxanthon-Tripletts, gemessen mit Nanosekundenlaserblitzspektroskopie (Anregung bei $355 \mathrm{~nm}$ ) anhand der Transientenabsorption bei $600 \mathrm{~nm}$. Für Verbindung 3 wurde kein Triplettsignal beobachtet.

Mit den Verbindungen 4 und 6 wurden bereits Experimente zum Aufbau von DNA-Chips durchgeführt. Unter optimierten Bedingungen ergaben sich 10-mal kürzere $\mathrm{Zy}$ kluszeiten als mit NPPOC-T $\left(\mathbf{1}, \mathrm{R}=5^{\prime}-O\right.$-Thymidinyl). Die Ausbeute pro Synthesezyklus lag bei ca. $90 \%$.

Um nachzuweisen, dass die Steigerung der Lichtempfindlichkeit auf eine intramolekulare Triplett-Energieübertragung zurückzuführen ist, wurden Nanosekundenlaserblitzspektroskopie-Experimente ausgeführt. Gemessen wurden Transientenabsorptionsspektren sowie die Abklingkurven des Thioxanthon-Triplettzustands. In der zeitlichen Folge der Entwicklung der Transientenabsorptionsspektren ließen sich das Abklingen der Triplett-Triplett-Absorption und das Anklingen der Absorption für die aci-Nitro-Form der
NPPOC-Gruppe nachweisen, die auch bei der nichtmodifizierten NPPOC-Verbindung als Intermediat der Schutzgruppenabspaltung auftritt. ${ }^{[10]}$

In stickstoffgesättigter Lösung sind die Triplettlebensdauern des Thioxanthons in allen bichromophoren Verbindungen wesentlich kürzer als diejenige von unsubstituiertem Thioxanthon (siehe Abbildung 2 und Tabelle 1). Darüber hinaus liegen die Quantenausbeuten von direkter und sensibilisierter Photoreaktion von NPPOC in ähnlicher Größenordnung. Diese beiden Beobachtungen belegen, dass die Triplettlöschung im Wesentlichen auf eine Energieübertragung zurückzuführen ist. Verbindung $\mathbf{6}$ unterscheidet sich in ihrem Transientenabsorptionsspektrum ebenso wie in ihrem Absorptionsspektrum (siehe Abbildung 1) wesentlich von allen anderen Thioxanthonen. Die beiden Chromophore koppeln stark und können daher nicht mehr separat betrachtet werden. Vermutlich wird die Lebensdauer des beobachteten Transienten auch in diesem System durch die HÜbertragung zu Beginn der Photoreaktion bestimmt, deren Quantenausbeute ähnlich hoch ist wie bei den ThioxanthonNPPOC-Verbindungen ohne direkte elektronische Kopplung.

In luftgesättigter Lösung wirkt molekularer Sauerstoff als Triplettlöscher und verringert in diffusionskontrollierter Reaktion die Triplettlebensdauer aller Thioxanthonverbindungen auf einen Wert im 100-ns-Bereich. Dieser Prozess konkurriert mit der Energieübertragung und verringert die Quantenausbeute der Schutzgruppenabspaltung. Ist die intramolekulare Energieübertragung schnell, oder hat der reagierende Triplettzustand ohnehin eine kurze Lebensdauer (wie bei Verbindung 6), dann hängt die Lichtempfindlichkeit wesentlich weniger vom Sauerstoffgehalt der Lösung ab. Bei den Photoreaktionen der Verbindungen 4-6 ist deshalb die Empfindlichkeit gegen molekularen Sauerstoff nur gering. Dieser Trend konnte auch bei den kontinuierlichen Bestrahlungen der neuen Schutzgruppenverbindungen in luftgesättigten Lösungen bestätigt werden. Insbesondere für den Einsatz im üblichen Herstellungsprozess von DNA-Chips ist dies ein entscheidender Vorteil, da ein Sauerstoff-freies Arbeiten einen erheblichen Aufwand erfordern würde.

Obwohl Triplett-Triplett-Energieübertragung in den neuen Verbindungen zweifelsfrei nachgewiesen wurde, muss festgestellt werden, dass die Sensibilisierung der photoinduzierten Schutzgruppen nicht allein über diesen Mechanismus verlaufen kann. Dies ergibt sich aus der Beobachtung von teilweise erheblicher Fluoreszenzlöschung, die mit einer

Tabelle 1: Spektroskopische und photochemische Kenndaten für 1-7. ${ }^{[a]}$

\begin{tabular}{|c|c|c|c|c|c|c|c|c|c|c|}
\hline \multirow[b]{2}{*}{ Verbindung } & \multicolumn{2}{|c|}{$\varepsilon\left[\mathrm{M}^{-1} \mathrm{~cm}^{-1}\right]$} & \multicolumn{2}{|c|}{$\phi$} & \multicolumn{2}{|c|}{$\varepsilon \phi\left[\mathrm{M}^{-1} \mathrm{~cm}^{-1}\right]$} & \multicolumn{2}{|c|}{ Thymidinausbeute } & \multicolumn{2}{|c|}{$\tau[\mu \mathrm{s}]$} \\
\hline & $366 \mathrm{~nm}$ & $405 \mathrm{~nm}$ & $366 \mathrm{~nm}$ & $405 \mathrm{~nm}$ & $366 \mathrm{~nm}$ & $405 \mathrm{~nm}$ & $366 \mathrm{~nm}$ & $405 \mathrm{~nm}$ & $\mathrm{~N}_{2}$-ges. & Luft-ges. \\
\hline 1 & 250 & $<40$ & 0.41 & - & 100 & $<40$ & 0.90 & - & {$[b]$} & {$[b]$} \\
\hline 2 & 3500 & 2700 & 0.42 & 0.38 & 1500 & 1000 & 0.21 & 0.33 & 1.05 & 0.18 \\
\hline 3 & 3100 & 3400 & 0.08 & 0.05 & 250 & 170 & 0.27 & 0.38 & {$[c]$} & {$[c]$} \\
\hline 4 & 3800 & 2300 & 0.21 & 0.14 & 800 & 320 & 0.86 & 0.75 & 0.11 & 0.08 \\
\hline 5 & 3900 & 2300 & 0.21 & 0.14 & 820 & 320 & 0.75 & 0.79 & 0.04 & 0.03 \\
\hline 6 & 8200 & 2400 & 0.26 & 0.27 & 2100 & 650 & 0.66 & 0.89 & 0.13 & 0.12 \\
\hline 7 & 4000 & 1800 & 0.09 & 0.09 & 360 & 160 & 0.92 & 0.82 & 3.6 & 0.22 \\
\hline
\end{tabular}

[a] Molarer dekadischer Absorptionskoeffizient $\varepsilon$, photochemische Quantenausbeute $\phi$, Lichtempfindlichkeitsmaß $\varepsilon \phi$, Ausbeute an freigesetztem Thymidin und Triplettlebensdauer $\tau$. [b] Die Triplettlebensdauern für das unsubstituierte Thioxanthon sind $22.2 \mu \mathrm{s}$ und $0.18 \mu \mathrm{s}$ in $\mathrm{N}_{2}$-gesättigtem bzw. luftgesättigtem $\mathrm{MeOH}$. [c] Es wurde kein Transient mit einer Lebensdauer im Nanosekundenbereich beobachtet. 
entsprechend reduzierten Bildung von Sensibilisator-Triplett einhergeht, sich jedoch kaum auf die photochemische Quantenausbeute auswirkt. Genauere Untersuchungen zum Mechanismus der Singulett-sensibilisierten Reaktion sind derzeit im Gange.

Unsere Ergebnisse lassen sich wie folgt zusammenfassen: Wir haben eine Reihe neuer photolabiler Schutzgruppen für die lichtgesteuerte DNA-Synthese mit sehr hoher Lichtempfindlichkeit hergestellt, in welchen die NPPOC-Schutzgruppe kovalent mit Thioxanthon als Antenneneinheit verknüpft ist. Die Photokinetik dieser Verbindungen unter stationären Bestrahlungsbedingungen wurde quantitativ untersucht, und die photochemischen Quantenausbeuten sowie die chemische Ausbeute der Substratfreisetzung wurden am Beispiel des Thymidins bestimmt. Der Triplett-Triplett-Energietransfer zwischen Antenneneinheit und reaktiver Schutzgruppe wurde mit Laserblitzspektroskopie nachgewiesen. Zusätzlich spielt bei kurzen Linkern jedoch eine Sensibilisierung über den angeregten Singulettzustand eine Rolle. Die hohe Lichtempfindlichkeit der neuen Schutzgruppen sollte den Zeitaufwand bei der photolithographischen Herstellung hochdichter DNA-Chips deutlich verringern.

Eingegangen am 17. November 2005

Online veröffentlicht am 23. März 2006

Stichwörter: DNA-Chips · Intramolekularer Energietransfer . Photochemie · Schutzgruppen · Sensibilisierung

[1] Einen ausgezeichneten Überblick hierzu liefert ein Themenheft: Nat. Genet. 1999, 21,(1), 1-60.

[2] K.-P. Stengele, U. E. Steiner, D. Wöll, W. Pfleiderer, S. Bühler, J. Bühler, R. Green, Abstr. Pap. Solid Phase Synthesis \& Combinatorial Libraries, London, United Kingdom, 2003; http://www. biocom.uk/sps2003.htm.

[3] NimbleGen Systems Inc., NimbleGen Releases First Human Whole-Genome Array, Pressemitteilung, Madison, WI, 28. Juli 2003; http://www.nimblegen.com/news/press_rel/news_2003 _07_28.html.
[4] S. P. A. Fodor, J. L. Read, M. C. Pirrung, L. Stryer, A. T. Lu, D. Solas, Science 1991, 251, 767-773.

[5] S. Singh-Gasson, R. D. Green, Y. Yue, C. Nelson, F. Blattner, M. R. Sussman, F. Cerrina, Nat. Biotechnol. 1999, 17, 974-978.

[6] „Photoremovable Protecting Groups in DNA Synthesis and Microarray Fabrication": M. C. Pirrung, V. S. Rana in Dynamic Studies in Biology: Phototriggers, Photoswitches, and Caged Compounds (Hrsg.: M. Goeldner, R. S. Givens), Wiley, New York, 2005, S. 341.

[7] a) G. H. McGall, A. D. Barone, M. Diggelmann, S. P. A. Fodor, E. Gentalen, N. Ngo, J. Am. Chem. Soc. 1997, 119, 5081-5090; b) A. C. Pease, D. Solas, E. J. Sullivan, M. T. Cronin, C. P. Holmes, S. P. A. Fodor, Proc. Natl. Acad. Sci. USA 1994, 91, $5022-5026$.

[8] Die Quantenausbeute für MeNPOC wurde unter den gleichen Bedingungen wie für NPPOC bestimmt $(\mathrm{MeOH}$, Bestrahlung bei $366 \mathrm{~nm}$ ).

[9] a) A. Hasan, K.-P. Stengele, H. Giegrich, P. Cornwell, K. R. Isham, R. A. Sachleben, W. Pfleiderer, R. S. Foote, Tetrahedron 1997, 53, 4247-4264; b) H. Giegrich, S. Eisele-Bühler, C. Hermann, E. Kvasyuk, R. Charubala, W. Pfleiderer, Nucleosides Nucleotides 1998, 17, 1987-1996; c) S. Bühler, I. Lagoya, H. Giegrich, K.-P. Stengele, W. Pfleiderer, Helv. Chim. Acta 2004, $87,620-659$.

[10] S. Walbert, W. Pfleiderer, U. E. Steiner, Helv. Chim. Acta 2001, $84,1601-1611$.

[11] D. Wöll, S. Walbert, K.-P. Stengele, T. J. Albert, T. Richmond, J. Norton, M. Singer, R. D. Green, W. Pfleiderer, U. E. Steiner, Helv. Chim. Acta 2004, 87, $28-45$.

[12] a) C. Sundararajan, D. E. Falvey, J. Am. Chem. Soc. 2005, 127, $8000-8001$; b) C. Sundararajan, D. E. Falvey, Org. Lett. 2005, 7 , 2631-2634; c) A. Banerjee, K. Lee, D. E. Falvey, Tetrahedron 1999, $55,12699-12710$.

[13] S. Speiser, Chem. Rev. 1996, 96, 1953-1976, zit. Lit.

[14] U. E. Steiner, Universität Konstanz, D. Wöll, DE 10315772 (A1), WO 2004089529, 2004 [Chem. Abstr. 2004, 141, 366034].

[15] G. Papageorgiou, M. Lukeman, P. Wan, J. E. T. Corrie, Photochem. Photobiol. Sci. 2004, 3, 366-373; G. Papageorgiou, D. Ogden, J. E. T. Corrie, J. Org. Chem. 2004, 69, 7228-7233.

[16] J. Smirnova, D. Wöll, W. Pfleiderer, U. E. Steiner, Helv. Chim. Acta 2005, 88, 891-904.

[17] G. Gauglitz, J. Photochem. 1976, 5, 41-47; G. Gauglitz, S. Hubig, J. Photochem. 1981, 15, 255-257. 